\title{
(WEAK) INCIDENCE BIALGEBRAS OF MONOIDAL CATEGORIES
}

\author{
ULRICH KRÄHMER and LUCIA ROTHERAY \\ Institut für Geometrie, Technische Universität Dresden, Dresden, Germany \\ e-mails: ulrich.kraehmer@tu-dresden.de; lucia.rotheray@tu-dresden.de
}

(Received 12 April 2019; revised 2 December 2019; accepted 3 February 2020; first published online 16 March 2020)

\begin{abstract}
Incidence coalgebras of categories in the sense of Joni and Rota are studied, specifically cases where a monoidal product on the category turns these into (weak) bialgebras. The overlap with the theory of combinatorial Hopf algebras and that of Hopf quivers is discussed, and examples including trees, skew shapes, Milner's bigraphs and crossed modules are considered.
\end{abstract}

2010 Mathematics Subject Classification. 16T10, 16 T05.

1. Introduction. Incidence coalgebras of categories, defined in [18], have been studied in several areas, notably Möbius inversion (see e.g., [21, 22, 23]) and combinatorial Hopf algebras (see e.g., $[\mathbf{9}, \mathbf{1 4}, \mathbf{1 5}, \mathbf{2 4}]$ ). The latter notion refers to Hopf algebras with a vector space basis indexed by a family of combinatorial objects (e.g., graphs or integer partitions), but the precise definition varies in the literature. The product and coproduct reflect unions and compositions of these objects; hence, the underlying coalgebra is (or is closely related to) an incidence coalgebra of a category $\mathcal{C}$.

In this case, one might wonder if the multiplication corresponds to a monoidal product on $\mathcal{C}$. This was explored by several authors, see [11] for a recent account. Here, we characterise two classes of monoidal categories giving rise to pointed, respectively, weak bialgebras:

THEOREM 1. If a monoidal product on a Möbius category $\mathcal{C}$ has the unique lifting of factorisation (ULF) property, then its linearisation turns the incidence coalgbera of $\mathcal{C}$ into a pointed bialgebra. This is a Hopf algebra provided that the monoid of objects is a group. Similarly, if $\mathcal{C}$ is a locally finite strict 2-group, the monoidal product turns the incidence coalgebra of $\mathcal{C}$ into a weak Hopf algebra.

See the main text for definitions.

A main goal of this paper is to discuss how several well-known examples of Hopf algebras fit into this picture, including the Connes-Kreimer Hopf algebra of rooted trees and symmetric functions. Milner's bigraphs, a combinatorial structure employed in theoretical computer science, are considered as a new example. We discuss how the Hopf algebraic techniques applied to the problem of renormalisation in physics could lead to new approaches to bigraphical systems, for example, in studying reaction rules, and it is our hope that there are other parallels to be drawn between bigraphical systems and physical ones, for example, Dyson-Schwinger type equations for generating sub-bialgebras.

We also show that not all Hopf algebras of a combinatorial nature can be described this way, even when the coalgebra structure is the incidence coalgebra of a category. As 
an illustrative, and perhaps counterintuitive, example we investigate the Hopf quivers of Cibils and Rosso:

THEOREM 2. If $Q=\left(Q_{0}, Q_{1}\right)$ is a Hopf quiver, $k$ a field and $\cdot: k Q \times k Q \rightarrow k Q$, the multiplication in the associated Hopf algebra $k \mathcal{C}$ as defined in [6], then $\cdot$ is the linear extension of a monoidal product on $Q$ if and only if $Q_{1}=\emptyset$.

The structure of the paper is as follows:

In Section 2, we recall some basic definitions, including that of an incidence coalgebra of a category, Möbius categories and the ULF property of a functor.

In Section 3, we study monoidal Möbius categories whose monoidal product is a ULF functor. We prove that these define bialgebras and discuss the examples mentioned above.

In Section 4, we recall the definition of a weak bialgebra and show that the monoidal product in a 2-group satisfies a weak version of the ULF property, which leads to the last statement in Theorem 1.

2. Definitions and notation. Throughout, all categories are assumed to be small and all monoidal categories to be strict. We use $\mathcal{C}$ to denote both a category and its set of morphisms, and $\mathcal{C}(x, y)$ the subset of morphisms from $x$ to $y$. We denote the set of objects by $O b \mathcal{C}$ and the set of identity morphisms by $I d \mathcal{C}$. The identity morphism at $x \in O b \mathcal{C}$ is written $i_{x}$. The monoidal product is denoted $:: \mathcal{C} \times \mathcal{C} \rightarrow \mathcal{C}$ to reserve $\otimes$ for the tensor product of vector spaces.

Definition 1 (Decompositions and length). Given a morphism $f \in \mathcal{C}$ and $n \in \mathbb{N}$, we define the set

$$
N_{n}(f):=\left\{\left(a_{1}, \ldots, a_{n}\right) \in \mathcal{C}^{\times n} \mid a_{1} \circ \ldots \circ a_{n}=f\right\},
$$

of $n$-decompositions of $f$, and let $\hat{N}_{n}(f)$ be the non-degenerate subset, that is, those decompositions for which no $a_{i}$ is an identity morphism. Furthermore, we set

$$
\hat{N}(f):=\bigcup_{n \in \mathbb{N}} \hat{N}_{n}(f), \quad \ell(f):=\sup \left\{n \mid \hat{N}_{n}(f) \neq \emptyset\right\},
$$

and call $\ell(f)$ the length of $f$.

Note that by definition, $N_{n}:=\bigcup_{f \in \mathcal{C}} N_{n}(f)$ is the set of $n$-simplices in the nerve of the category $\mathcal{C}$. This leads to the more topological viewpoint of [11].

DEFINITION 2 (Locally finite and Möbius categories). A category $\mathcal{C}$ is called locally finite, respectively, Möbius, if $\left|N_{2}(f)\right|$, respectively $|\hat{N}(f)|$, is finite for every $f \in \mathcal{C}$.

These concepts appear under various names in the literature (in particular, locally finite categories are also called finely finite, for example, by [23]); we follow the terminology of Joni and Rota [18] and Leroux [22] (see also [21]). Evidently, a Möbius category does not contain nontrivial isomorphisms or idempotents.

LEMMA 1. A category $\mathcal{C}$ is Möbius if and only if $\mathcal{C}$ is locally finite and every morphism has finite length.

For the proof, see [22] or [21, Proposition 2.8].

Note that the sequence of sets

$$
\mathcal{C}_{n}:=\{f \in \mathcal{C} \mid \ell(f) \leq n\},
$$

is a filtration of $\mathcal{C}$, called the length filtration. If $\mathcal{C}$ is Möbius, then this filtration is exhaustive and $\mathcal{C}$ is one-way, that is, $\mathcal{C}_{0}=I d \mathcal{C}$. 
The following definition is originally due to Joni and Rota [18]; we consider a variation in which the coproduct is multiplied by a nonzero scalar, as this rescaling arises naturally from the compatibility with the associative products discussed below:

DEFINITION 3 ((Scaled) incidence coalgebra of a category). Let $\mathcal{C}$ be a locally finite category, $k$ a field, $k \mathcal{C}$ the $k$-vector space spanned by the morphisms of $\mathcal{C}$ and $\lambda \in k, \lambda \neq 0$. The coassociative counital coalgebra structure on $k \mathcal{C}$ defined by the following formula is called the (scaled) incidence coalgebra of $\mathcal{C}$ over $k$ :

$$
\Delta(f):=\frac{1}{\lambda} \sum_{(a, b) \in N_{2}(f)} a \otimes b, \quad \varepsilon(f):=\left\{\begin{array}{l}
\lambda, f \in I d \mathcal{C}, \\
0, f \notin I d \mathcal{C} .
\end{array}\right.
$$

We will combine such coalgebra structures on $k \mathcal{C}$ with the algebra structure defined by a monoidal product on $\mathcal{C}$ :

LEMMA 2. If $(\mathcal{C}, \cdot, 1)$ is a monoidal category, $k$ is a field and $k \mathcal{C}$ is the $k$-vector space spanned by $\mathcal{C}$, then $\left(k \mathcal{C}, \cdot, i_{1}\right)$ is a unital associative $k$-algebra.

Next, we address the question of whether these algebra and coalgebra structures define a bialgebra, that is, whether $\Delta$ and $\varepsilon$ are algebra morphisms. We use the following definitions:

Definition 4 ( $n$-to-1 surjection). A map $\phi: A \rightarrow B$ is an $n$-to-1 surjection if the preimage of every element of $B$ has cardinality $n$.

DEFINITION 5 (Lifting of factorisations property). For $n \in \mathbb{N}$, a functor $F: \mathcal{C} \rightarrow \mathcal{D}$ has the $n$-to-1 lifting of factorisations property if the map

$$
\begin{gathered}
N_{2}(f) \rightarrow N_{2}(F f) \\
(a, b) \mapsto(F a, F b),
\end{gathered}
$$

is an $n$-to- 1 surjection for all $f \in \mathcal{C}$.

This generalises the ULF property defined, for example, in $[\mathbf{1 1}, \mathbf{1 2}, \mathbf{2 1}]$. For consistency with the literature, we will use the name ULF rather than 1LF. The generalised concept will be used in the final section of this paper in the construction of weak bialgebras.

LEMMA 3. If $F: \mathcal{C} \rightarrow \mathcal{D}$ has the ULF property, then $F$ reflects identities, that is, $F f \in$ $I d \mathcal{D} \Leftrightarrow f \in I d \mathcal{C}$.

Proof. $f \in I d \mathcal{C} \Rightarrow F f \in I d \mathcal{D}$ holds as $F$ is a functor. $F f \in I d \mathcal{D} \Rightarrow f \in I d \mathcal{C}$ follows from the injectivity of the map $N_{2}(f) \rightarrow N_{2}(F f)$.

3. Bialgebras from monoidal categories. In this section, we study monoidal Möbius categories for which the monoidal product turns the incidence coalgebra into a bialgebra.

Definition 6 (Combinatorial category). A combinatorial category is a monoidal Möbius category $(\mathcal{C}, \cdot, 1)$ whose product $:: \mathcal{C} \times \mathcal{C} \rightarrow \mathcal{C}$ has the ULF property.

THEOREM 3. Let $\mathcal{C}$ be a locally finite category, $k$ a field, and $\left(k \mathcal{C}, \cdot, i_{1}\right)$ and $(k \mathcal{C}, \Delta, \varepsilon)$ be the (co)algebras defined in Lemma 2 and Defintion 3, respectively (with $\lambda=1$ ). 
(1) If $\mathcal{C}$ is a Möbius category, then the coalgebra $k \mathcal{C}$ is pointed, that is, its simple subcoalgebras are one-dimensional as $k$-vector spaces.

(2) If $\mathcal{C}$ is a combinatorial category, then $\left(k \mathcal{C}, \cdot, i_{1}, \Delta, \varepsilon\right)$ is a k-bialgebra, that is, $\Delta$ and $\varepsilon$ are algebra morphisms.

(3) IfC is a combinatorial category, then the (two-sided) ideal $I \triangleleft k \mathcal{C}$ generated by

$$
\left\{i_{x}-i_{1} \mid x \text { is not invertible in }(O b \mathcal{C}, \cdot, 1)\right\},
$$

is a coideal, and the bialgebra $\mathrm{kC} / \mathrm{I}$ is a pointed Hopf algebra.

For the proof, we will use the following results:

LEMMA 4. If $k$ is a field, $C$ is a $k$-coalgebra and $\left\{C_{n}\right\}_{n=0}^{\infty}$ is a (exhaustive) filtration of $C$, then any simple subcoalgebra of $C$ is contained in $C_{0}$.

LEMMA 5. If $k$ is a field and B is a pointed k-bialgebra, then B is a Hopf algebra if and only if the set of group-like elements of $B$ is a group under multiplication.

Lemma 6. Let $f: C \rightarrow D$ be a surjective coalgebra homomorphism. Any simple subcoalgebra $F \subseteq D$ is contained in $f(E)$ for some simple subcoalgebra $E \subseteq C$.

See [27, Proposition 4.1.2, Lemma 7.6.3 and Proposition 4.1.7] for the proofs.

Proof of Theorem 3. (1) As $\mathcal{C}$ is Möbius, the length filtration is an exhaustive coalgebra filtration and $\mathcal{C}$ is one-way, so that $k \mathcal{C}_{0}$ is spanned by the monoid $\left(\operatorname{IdC}, \cdot i_{1}\right) \cong$ $(O b \mathcal{C}, \cdot, 1)$ whose elements are all group-like, $\Delta\left(i_{x}\right)=i_{x} \otimes i_{x}$. This coalgebra is evidently pointed, and by Lemma 4 , all simple subcoalgebras of $k \mathcal{C}$ are contained in $k \mathcal{C}_{0}$. Consequently, $k \mathcal{C}$ itself is pointed as well.

(2) As $\mathcal{C}$ is a Möbius category, the unit element $i_{1}$ of the associative algebra $(k \mathcal{C}, \cdot)$ is group-like, that is, $\Delta\left(i_{1}\right)=i_{1} \otimes i_{1}$, which means that $\Delta$ is unital. The ULF property of the product - further ensures that the coproduct $\Delta$ is also multiplicative:

$$
\begin{aligned}
\Delta(f \cdot g) & =\sum_{\left(h_{1}, h_{2}\right) \in N_{2}(f \cdot g)} h_{1} \otimes h_{2} \\
& =\sum_{\left(\left(f_{1}, f_{2}\right),\left(g_{1}, g_{2}\right)\right) \in N_{2}(f) \times N_{2}(g)} f_{1} \cdot g_{1} \otimes f_{2} \cdot g_{2} \\
& =\Delta(f) \cdot \Delta(g) .
\end{aligned}
$$

Hence, $\Delta$ is a morphism of unital algebras. The unit element $i_{1}$ is an identity morphism, so $\varepsilon\left(i_{1}\right)=1$. As ULF functors reflect identities (Lemma 3), we have

$$
\varepsilon(f \cdot g)=\left\{\begin{array}{l}
1, f \cdot g \in I d \mathcal{C} \Leftrightarrow f, g \in I d \mathcal{C} \\
0 \text { else }
\end{array}=\varepsilon(f) \varepsilon(g),\right.
$$

and hence $\varepsilon$ is also a morphism of unital algebras.

(3) That $I$ is a coideal follows from

$$
\Delta\left(i_{x}-i_{1}\right)=\left(i_{x}-i_{1}\right) \otimes i_{x}+i_{1} \otimes\left(i_{x}-i_{1}\right),
$$

and the multiplicativity of $\Delta$. Denote by $Q:=k \mathcal{C} / I$ the quotient bialgebra and by $q: k \mathcal{C} \rightarrow Q$ the quotient map. The length filtration on $k \mathcal{C}$ induces an exhaustive filtration on $Q$, that is, $Q_{n}:=q\left((k \mathcal{C})_{n}\right)$. Any simple subcoalgebra of $Q$ is the image of a simple subcoalgebra of $k \mathcal{C}$ (Lemma 6) and therefore, as $k \mathcal{C}$ is pointed, has 
dimension 1. Hence, $Q$ is pointed. The claim that $Q$ is a Hopf algebra then follows by application of Lemma 5 .

DEFINITION 7 (Incidence bialgebra). We call $\left(k \mathcal{C}, \cdot, i_{1}, \Delta, \varepsilon\right)$ the incidence bialgebra of the combinatorial category $\mathcal{C}$ over the field $k$.

REMARK 1. As well as the Hopf algebra $Q:=k \mathcal{C} / I$ from Theorem 3 (3), one can take further Hopf algebra quotients. For example in [19, Section 4.4.2], it is observed that the quotient of $k \mathcal{C}$ by the bialgebra ideal generated by all relations $i_{x}-i_{1}$ (which contains $I$ ) is a Hopf algebra. In Sections 3.3 and 3.4, we will see that some well-studied Hopf algebras, namely those of symmetric functions and rooted trees, can be realised as quotient Hopf algebras of a incidence bialgebras.

The remainder of this section is devoted to the discussion of some examples of combinatorial categories and their incidence bialgebras.

3.1. Example: The thin case. If $(M, \preccurlyeq)$ is a preordered set, that is, $\preccurlyeq$ is a reflexive and transitive binary relation on $M$, then

$$
\mathcal{C}_{M}(y, x):=\left\{\begin{array}{ll}
\{(x, y)\} & x \preccurlyeq y \\
\varnothing & x \npreceq y
\end{array},\right.
$$

is a category with $O b \mathcal{C}_{M}:=M$. So an element $(x, y) \in \mathcal{C}_{M} \subseteq M \times M$ is the (unique) morphism $y \rightarrow x$, and composition and identity morphisms are given by

$$
(x, y) \circ(y, z):=(x, z), \quad i_{x}=(x, x) .
$$

In this way, preorders correspond bijectively to thin categories (categories with at most one morphism between any two given objects).

Proposition 1. The category $\mathcal{C}_{M}$ is locally finite if and only if all intervals

$$
[x, y]:=\{z \in M \mid x \preccurlyeq z \preccurlyeq y\},
$$

are finite. It is Möbius if and only if it is locally finite and $\preccurlyeq$ is a partial ordering.

Proof. The first statement follows directly from the defintion. For the second, note that $\mathcal{C}_{M}$ is by definition Möbius if and only if for all $x, y \in M,|[x, y]|<\infty$ and there exists $\ell(x, y) \in \mathbb{N}$ such that (with $a \prec b$ meaning $a \preccurlyeq b \wedge a \neq b$ )

$$
\forall z_{1}, \ldots, z_{l} \in M: x=z_{1} \prec z_{2} \prec \ldots \prec z_{l}=y \quad \Rightarrow \quad l \leq \ell(x, y) .
$$

If $\preccurlyeq$ is a partial ordering, then it is immediate that the elements $z_{i}$ in such chains are pairwise distinct; hence, the length $l$ of such chains is bounded by $|[x, y]|<\infty$. If $\preccurlyeq$ is conversely not a partial ordering, then there exist $x, y \in M$ with $x \prec y, y \prec x$, and in this case, there are arbitrarily long chains of the form

$$
x \prec y \prec x \prec y \prec \ldots
$$

Assume now that $(M, \preccurlyeq, \cdot)$ is a preordered monoid, that is,

$$
x \preccurlyeq y, z \preccurlyeq t \Rightarrow x \cdot z \preccurlyeq y \cdot t,
$$

that holds for all $x, y, z, t \in M$. This corresponds to $\mathcal{C}_{M}$ becoming monoidal via

$$
(x, y) \cdot(z, t):=(x \cdot z, y \cdot t) .
$$


The condition (3.1) also means that the set of all nonempty intervals $[x, y]$ inherits a monoid structure from $M$; note that this is a quotient monoid of $\mathcal{C}_{M}$ via

$$
f=(x, y) \mapsto[f]:=[x, y] .
$$

By definition, we have

Proposition 2. The monoidal product $\cdot$ on $\mathcal{C}_{M}$ is $U L F$ if and only if the map

$$
\left(\mathcal{C}_{M}, \cdot, i_{1}\right) \rightarrow\left(\mathbb{N}_{+}, \cdot, 1\right), \quad f \mapsto|[f]|,
$$

is a monoid morphism to the multiplicative monoid of positive natural numbers.

3.2. Example: Quotients of monoids. When a preorder $\preccurlyeq$ is an equivalence relation, then the interval $[x, y]$ is simply the equivalence class $[x]$ of $x$ (and of $y$ ); hence, $\mathcal{C}_{M}$ is locally finite if and only if all equivalence classes are finite. In this case, the incidence coalgebra of $\mathcal{C}_{M}$ is a direct sum of matrix coalgebras (and is in particular a cosemisimple coalgebra),

$$
k \mathcal{C}_{M} \cong \bigoplus_{[x] \in M / \preccurlyeq} M_{|[x]|}(k) .
$$

Note that $\mathcal{C}_{M}$ is not Möbius unless $\preccurlyeq$ is $=$, as any $(x, y)$ with $x \prec y$ is a nontrivial ismorphism.

As a concrete example, consider the free monoid $S$ in two generators 0,1 :

DEFINITION 8 (Monoid of paths). For $n \in \mathbb{N}$, a path of length $n$ is a word of length $n$ in the alphabet $\{0,1\}$. We denote the set of all paths by $S$. For two paths $q=q_{1} \ldots q_{n}$ and $p=p_{1} \ldots p_{m} \in S$, we define the product $q \cdot p$ to be the word

$$
q \cdot p=q_{1} \ldots q_{n} p_{1} \ldots p_{m} .
$$

We represent $p \in S$ by a path in the real plane starting at $(0,0)$ and composed of horizontal steps $(1,0)$ and vertical steps $(0,1)$. We take a horizontal step at each letter $p_{i}=0$ and a vertical step at each letter $p_{i}=1$ :

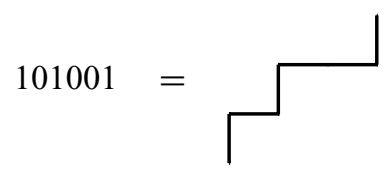

Now call two paths equivalent if they have the same length,

$$
q_{1} \cdots q_{m} \sim p_{1} \cdots p_{n}: \Leftrightarrow m=n .
$$

Then, $(S, \sim)$ is a preordered monoid and $k \mathcal{C}_{S}$ is the free algebra on four generators $\alpha=$ $(0,0), \beta=(0,1), \gamma=(1,0)$ and $\delta=(1,1)$. The map (3.2) is given by

$$
\left(p_{1} \cdots p_{n}, q_{1} \cdots q_{n}\right) \mapsto 2^{n},
$$

so $\cdot$ is ULF. The coproduct of the generators is given by

$$
\begin{array}{ll}
\Delta(\alpha)=\alpha \otimes \alpha+\beta \otimes \gamma, & \Delta(\beta)=\alpha \otimes \beta+\beta \otimes \delta, \\
\Delta(\gamma)=\gamma \otimes \alpha+\delta \otimes \gamma, & \Delta(\delta)=\gamma \otimes \beta+\delta \otimes \delta .
\end{array}
$$

So $k \mathcal{C}_{S}$ is a bialgebra (although $\mathcal{C}_{S}$ is not Möbius), but it is not a Hopf algebra. 
3.3. Example: Partially ordered monoids. Recall that for a partially ordered monoid, $\mathcal{C}_{M}$ is Möbius and equal to the monoid of intervals in $M$. Any preorder $\preccurlyeq$ defines an equivalnce relation $x \sim y: \Leftrightarrow x \preccurlyeq y \wedge y \preccurlyeq x$ and induces a partial ordering on $M / \sim$. If $M$ is a preordered monoid, then $M / \sim$ becomes a partially ordered monoid. Hence, any preordered monoid has a canonical reduced version which is partially ordered.

Incidence bialgebras of intervals in parially ordered monoids have been extensively studied in the literature. Here, we will focus on the specific example of skew shapes and discuss Hopf algebra quotients of their incidence bialgebra.

DEFINITION 9 (Partial ordering on paths). Let $S$ be the monoid of paths from Defintion 8. For a path $p=p_{1} \ldots p_{n} \in S$, we define the height of $p$ as the integer $h(p):=$ $\sum_{i} p_{i}$ and the width of $p$ as the integer $w(p):=n-h(p)$. For two paths $q, p \in S$ with $h(p)=h(q)$ and $w(p)=w(q)$, we define

$$
q \leq p: \Leftrightarrow \sum_{j=1}^{i} p_{j} \geq \sum_{j=1}^{i} q_{j} \forall i=1, \ldots, n .
$$

In this way, $S$ becomes a partially ordered monoid.

DEFINITION 10 (Category of skew shapes). We denote by $\mathcal{S}:=\mathcal{C}_{S}$ the monoidal Möbius category defined by the partially ordered monoid $(S, \leq, \cdot)$. A morphism $(q, p) \in \mathcal{S}$ will be referred to as a skew shape.

The skew shape $(q, p)$ will be represented by drawing all paths $r \in[q, p]$.

EXAMPLE 1. If

$$
r=00101, q=01010, p=10100,
$$

then the skew shapes

$$
(r, q) \circ(q, p)=(r, p), \quad(q, p) \cdot(r, q)=(q \cdot r, p \cdot q),
$$

will be depicted as follows:

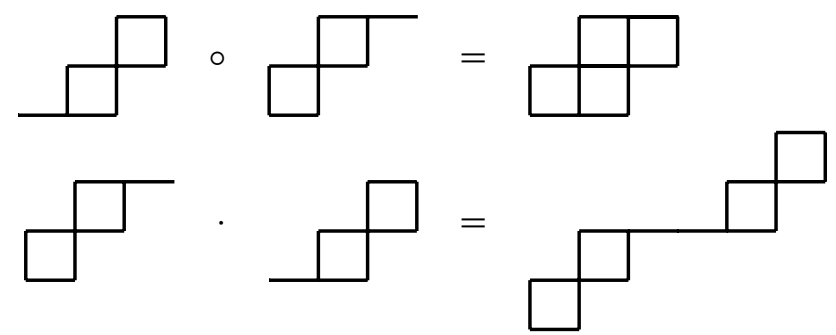

The graphical representation makes it evident that the monoid $(\mathcal{S}, \cdot)$ is free on the set

$$
\Gamma:=\left\{(q, p) \in \mathcal{S} \mid \sum_{j=1}^{i} p_{j}>\sum_{j=1}^{i} q_{j} \forall i=1, \ldots, h(p)+w(p)-1\right\} .
$$

In the above Example 1, we have $(r, p) \in \Gamma$ and

$$
(q, p)=\alpha \cdot \alpha \cdot \beta, \quad(r, q)=\beta \cdot \alpha \cdot \alpha, \quad \alpha:=(01,10), \beta:=(0,0) \in \Gamma .
$$

The category $\mathcal{S}$ is combinatorial, so by Theorem 3 there exists an incidence bialgebra structure on the vector space $k \mathcal{S}$. 
EXAMPLE 2. For the shape $(r, q)$ as in Example 1, the coproduct in $k \mathcal{S}$ is given by

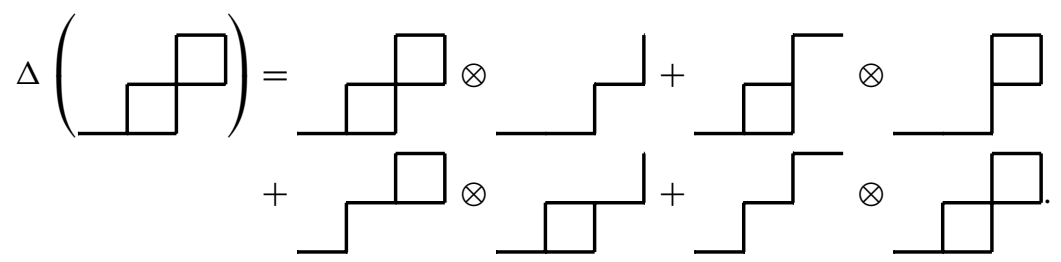

REMARK 2 (Quotients of $k \mathcal{S} / I$ ). By Theorem 3(3), the ideal $I \triangleleft k \mathcal{S}$ generated by the elements $\{(0,0)-1,(1,1)-1\}$ is a coideal and $k \mathcal{S} / I$ is a Hopf algebra. There are three further quotients of $k \mathcal{S} / I$ which are well-known Hopf algebras:

(1) The Hopf algebra of skew shapes [30] is the abelianisation of $k \mathcal{S} / I$ (i.e., the quotient by the ideal generated by the elements $\{\mu \cdot v-v \cdot \mu \mid \mu, v \in k \mathcal{S} / I\})$.

(2) The Hopf algebra $k \Lambda$ of symmetric functions: if for $\gamma \in \Gamma \backslash\{(0,0),(1,1)\}$, we let $s_{\gamma}$ denote the (skew) Schur function on $\gamma$ [14, Definitions 2.8,2.19], then the map

$$
k \mathcal{S} / I \rightarrow k \Lambda, \quad\left(\gamma_{1}+I\right) \cdots\left(\gamma_{m}+I\right) \mapsto \prod_{i=1}^{m} s_{\gamma_{i}},
$$

is a surjective Hopf algebra homomorphism.

3.4. Example: Rooted forests and the Connes-Kreimer Hopf algebra. The example we consider here is a combinatorial category that is not thin. Its incidence bialgebra has been studied by various authors recently (it appears explicitly in $[9,19,20,25]$ and is used implicitly in $[\mathbf{2}, \mathbf{2 8}]$ ) due to its close relation to the Connes-Kreimer Hopf algebra [7].

DEFINITION 11 (Operadic rooted forest). An operadic rooted forest consists of:

(1) A finite poset $(f, \preccurlyeq)$ in which all half intervals $(-\infty, y]:=\{x \in f \mid x \preccurlyeq y\}$ are totally ordered (the rooted forest). A minimal element is called a root, and the set of all these is denoted by $R(f)$. For each $x \in f$, we call

$$
C(x):=\{y \in f||[x, y] \mid=2\},
$$

the set of children of $x$.

(2) A chosen set $L(f) \subset f$ of maximal elements called the set of leaves.

We denote by $r_{f}: L(f) \rightarrow R(f)$ the unique map with $r_{f}(x) \preccurlyeq x$ for all $x \in L(f)$.

DEFINITION 12 (Operadic planar rooted forest). An operadic planar rooted forest is an operadic rooted forest with a total ordering of $R(f)$ and of each set $C(x)$.

Operadic planar rooted forests can be visualised as planar graphs with vertex set $f$ and with the roots drawn at the top. The total orderings of $R(f)$ and of the $C(x)$ are represented by the order of the vertices from left to right. Leaves are drawn as white vertices, and all other elements of $f$ as black vertices.

EXAMPLE 3. The following planar graphs represent planar rooted forests with $|f|=$ $3,|R(f)|=|L(f)|=1$ and $|g|=6,|R(g)|=1,|L(g)|=2$ :
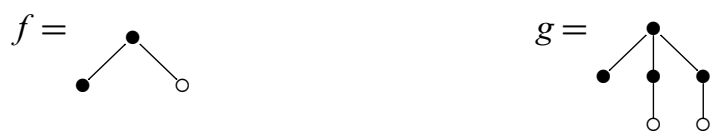
DEFINITION 13 (Category of operadic planar rooted forests). The set $\mathcal{C}_{R F}(m, n)$ of isomorphism classes of operadic planar rooted forests with $n$ roots and $m$ leaves is the set of morphisms in a combinatorial category $\mathcal{C}_{R F}$ with $\left(O b \mathcal{C}_{R F}, \cdot\right)=(\mathbb{N},+)$. For $f_{1} \in \mathcal{C}_{R F}(m, n)$ and $f_{2} \in \mathcal{C}_{R F}(p, m), f_{1} \circ f_{2}$ is the forest obtained by identifying the roots of $f_{2}$ with the leaves of $f_{1}$ as dictated by their total order. The monoidal product $f_{1} \cdot f_{2}$ is the ordered sum (noncommutative disjoint union) in which all vertices (previously) in $f_{2}$ are greater than all vertices (previously) in $f_{1}$.

EXAMPLE 4. With $f, g$ as in Example 3, we have
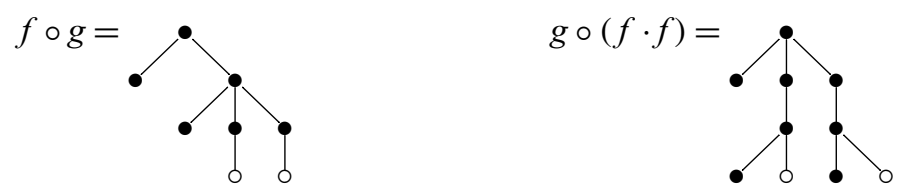

The category $\mathcal{C}_{R F}$ is combinatorial, so by Theorem 3 there exists an incidence bialgebra structure on the vector space $k \mathcal{C}_{R F}$.

EXAMPLE 5. For the forest $f$ as in Example 3, the coproduct in $k \mathcal{C}_{R F}$ is given by

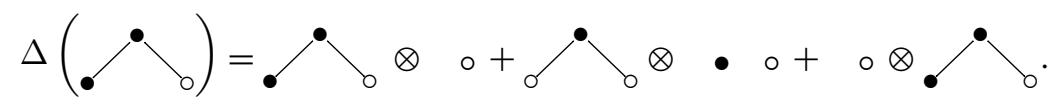

REMARK 3. Theorem 3(3) yields a Hopf algebra $k \mathcal{C}_{R F} / I$. Visually, this deletes all identities, that is, trees with no black vertices. As for the category of skew shapes, probably the most studied Hopf algebra associated to rooted trees (the Connes-Kreimer Hopf algebra) is a further quotient.

DEFINITION 14 (Core). The core of a planar rooted forest $f$ is the planar rooted forest $\operatorname{core}(f):=f \backslash L(f)$ (with the induced relations $\preccurlyeq, \leq$ and $L(\operatorname{core}(f)):=\emptyset$ ).

EXAMPLE 6. With $f, g$ as in Example 3, we have

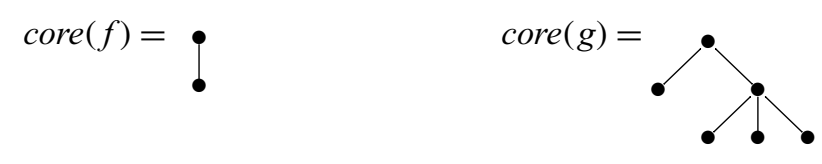

In particular, note that for any $i_{n} \in I d \mathcal{C}_{R F}, \operatorname{core}\left(i_{n}\right)=i_{1}=\emptyset$. Let $H_{P}$ denote the Hopf algebra of planar rooted trees [9]. This is a non-commutative version of the ConnesKreimer Hopf algebra $H_{C K}$ of rooted trees [7] and can be viewed as a further quotient of $k \mathcal{C}_{R F} / I$ by the ideal generated by all elements of the form $\{f-g \mid \operatorname{core}(f)=\operatorname{core}(g)\}$. See [20, Section 7] for further discussion.

3.5. Example: A toy model for bigraphs. Bigraphs (see $[\mathbf{1 7}, \mathbf{2 6}, \mathbf{2 9}]$ ) are combinatorial objects originally developed in theoretical computer science to model mobile computation. Bigraphs, as defined by Milner, describe the morphisms of a monoidal precategory [26, Section 2.2-2.3], that is, a monoidal category in which not all compositions or monoidal products are defined. We work with a restricted definition of bigraphs, which allows us to define a combinatorial category. This toy model admits the basic bigraph operations (composition, reactions and reductions) but does not include all features. For 
example, we only consider private names for bigraphs and essentially work in the support quotient of Milner's pre-category.

As the name suggests, a bigraph consists of two graphs. The first is almost an operadic planar rooted tree, but the ordering of the internal vertices is lost:

DEFINITION 15 (Place graph). A place graph is an operadic rooted forest $f$ together with total orderings of its roots and leaves that are compatible in the sense that the root map $r_{f}: L(f) \rightarrow R(f)$ is non-decreasing.

In the bigraph literature, the leaves of a place graph are called sites.

Definition 16 (Link graph). A link graph is a triple $(P, X, Y)$ of finite disjoint sets, together with total orders of $X$ and $Y$ and an equivalence relation $\sim$ on $P \cup X \cup Y$. Each equivalence class must contain at least two elements and cannot be entirely contained in $X$ or $Y$.

The elements of $P, X$ and $Y$ are called ports, inner names and outer names, respectively.

DEFINITION 17 (Bigraph). A bigraph $g=\left(g_{p}, g_{l}, \rho\right)$ consists of

(1) A place graph $g_{p}$.

(2) A link graph $g_{l}=(P, X, Y, \sim)$.

(3) A map $\rho: P \rightarrow g_{p} \backslash\left(L\left(g_{p}\right) \cup R\left(g_{p}\right)\right)$.

The pairs $\left(\left|L\left(g_{p}\right)\right|,|X|\right)$ and $\left(\left|R\left(g_{p}\right)\right|,|Y|\right)$ are called the inner and outer interfaces of $g$.

We visualise bigraphs as follows: roots and sites are represented by boxes in the plane, vertices by circles and ports by dots. The place graph structure $\preccurlyeq$ is indicated by nesting, the map $\rho$ by placing a port directly on its associated vertex and the equivalence relation $\sim$ by lines joining equivalent elements.

EXAMPLE 7. The bigraph with data

$$
\begin{aligned}
& g_{p}=\left\{r_{0}, v_{0}, s_{0}, s_{1}\right\}, \quad R\left(g_{p}\right)=\left\{r_{0}\right\}, \quad L\left(g_{p}\right)=\left\{s_{0}, s_{1}\right\}, \quad s_{0} \prec r_{0}, s_{1} \prec v_{0} \prec r_{0}, \\
& X=\left\{x_{0}, x_{1}\right\}, \quad Y=\emptyset, \quad P=\left\{p_{0}, p_{1}\right\}, \quad x_{0} \sim p_{0}, x_{1} \sim p_{1}, \quad \rho\left(p_{0}\right)=\rho\left(p_{1}\right)=v_{0}
\end{aligned}
$$

will be drawn as:

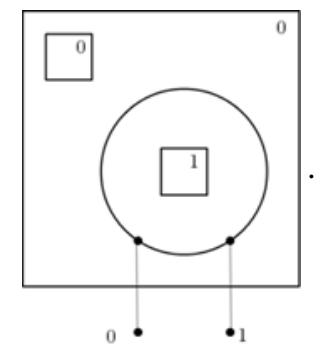

DEFINITION 18 (Category of bigraphs). We consider a category $\mathcal{C}_{B}$ with $O b \mathcal{C}_{B}=\mathbb{N} \times$ $\mathbb{N}$ and $\mathcal{C}_{B}((m, x),(n, y))$ the set of isomorphism classes of bigraphs with inner and outer interfaces $(m, x)$ and $(n, y)$, respectively. The composition of two bigraphs $g=\left(g_{p}, g_{l}, \rho_{g}\right)$ and $f=\left(f_{p}, f_{l}, \rho_{f}\right)$ is given as follows:

(1) The composition of the place graphs is defined analogously to that of operadic planar rooted trees, but one also deletes the roots of $f_{p}$. So the set underlying $g_{p} \circ f_{p}$ is $\left(g_{p} \dot{\cup} f_{p}\right) \backslash\left(R\left(f_{p}\right) \dot{\cup} L\left(g_{p}\right)\right)$. 
(2) The composition of the two link graphs $g_{l}=\left(P_{1}, X_{1}, Y_{1}, \sim_{1}\right)$ and $f_{l}=$ $\left(P_{2}, X_{2}, Y_{2}, \sim_{2}\right)$ is given by $g_{l} \circ f_{l}:=\left(P_{1} \oplus P_{2}, X_{1}, Y_{2}, \sim\right)$, where $\oplus$ is the ordinal sum and $\sim$ is generated by $\sim_{1}$ and $\sim_{2}$ (including $x \sim y$ if $x \in X_{1} \cup P_{1}, y \in P_{2} \cup Y_{2}$ and $x \sim_{1} z \sim_{2} y$ for some $z \in Y_{1}=X_{2}$ ).

(3) The ports stay assigned to the same vertices as before by the map $\rho_{g} \dot{\cup} \rho_{f}$.

The monoidal product of $g$ and $f$ is given componentwise by ordered unions.

We denote the identity at the interface $(m, x)$ by $i_{m, x}$. The monoid $\left(\operatorname{IdC}_{B}, \cdot\right)$ is freely generated by the set $\left\{i_{0,1}, i_{1,0}\right\}=\left\{{ }^{\circ}, \square^{0}, \square\right\}$.

EXAMPLE 8 (Composition and product). Consider the bigraphs
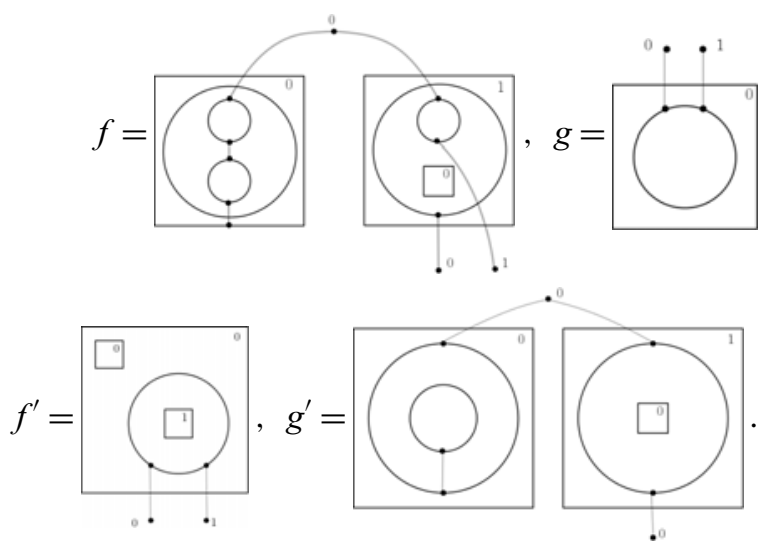

We have
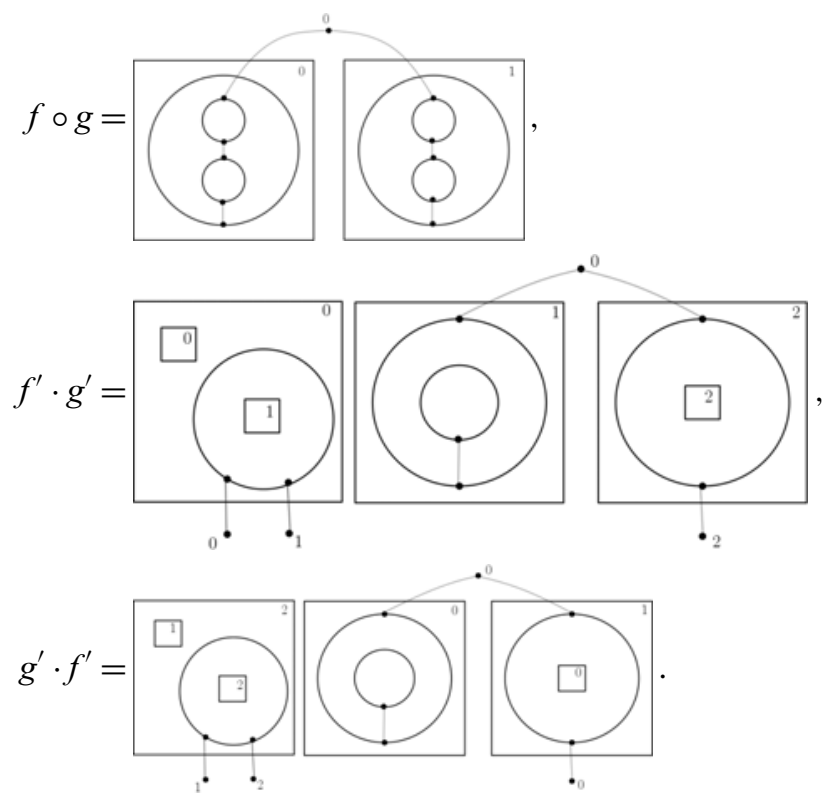

This category is combinatorial. So, by Theorem 3 , it defines an incidence bialgebra $k \mathcal{C}_{B}$. 
EXAMPLE 9 (Coproduct in $k \mathcal{C}_{B}$ ). For the bigraph $f^{\prime}$ from the previous example, the coproduct in $k \mathcal{C}_{B}$ is given by

$$
\Delta\left(f^{\prime}\right)=f^{\prime} \otimes i_{2,2}+i_{1,0} \otimes f^{\prime}+\square \square .
$$

REMARK 4. We hope that the Hopf algebraic techniques used to study $\mathcal{C}_{R T}$ and $H_{C K}$ will provide new approaches to studying bigraphical systems. In particular, one possible application is in the study of reaction rules. Put simply, a reaction rule is a map $r: \mathcal{C}_{B} \rightarrow \mathcal{C}_{B}$ which removes a certain subset of bigraphs by mapping them to simpler ones. These rules are not necessarily compatible with the composition, that is, in general, we have $r(g \circ f) \neq$ $r(g) \circ r(f)$.

EXAMPLE 10. Consider the reaction rule for a version of $\mathcal{C}_{B}$ in which vertices can be labelled by $\{A, B, C\}$ :

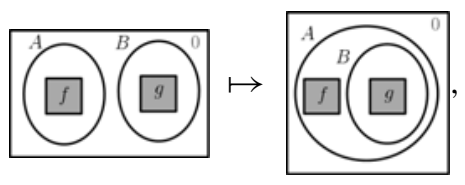

where the grey boxes $f, g$ denote any substructure. This rule demands that the $A$ and $B$ vertices in the initial graph are siblings (i.e., in some set $C(x)$ ). This means that $r$ maps the graphs

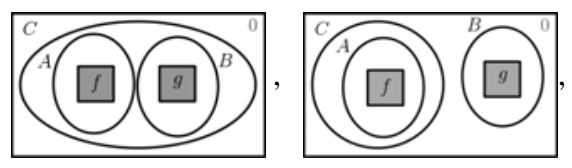

to

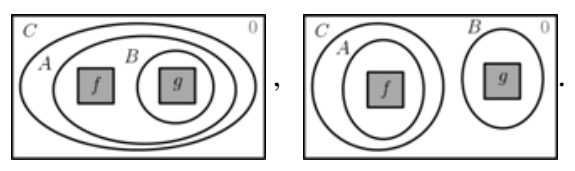

The second graph shows no reaction even though the bigraph does contain the relevant subgraph.

The coproduct allows us to see such blocked reactions. For our example, define the new map

$$
r^{\prime}:=(\mathrm{id} \otimes r M \circ) \Delta: k \mathcal{C}_{B} \rightarrow k \mathcal{C}_{B} \otimes k \mathcal{C}_{B}
$$

where

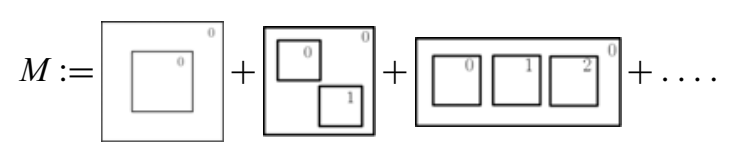


Applying this, we have

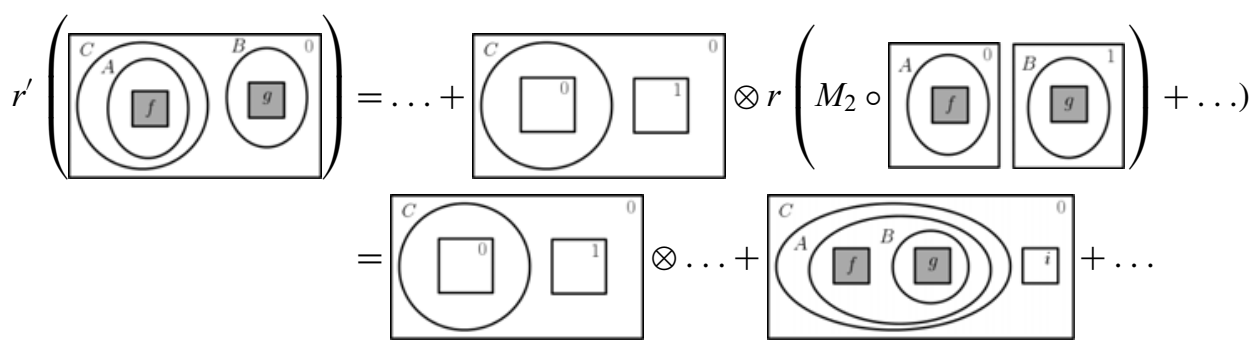

where $i \in \mathbb{N}$ is determined by the interfaces of $f$ and $g$.

3.6. A notable non-example: Hopf quivers. We now consider an example of an incidence coalgebra which admits a multiplication making it a Hopf algebra but is not (generally) an example of our construction.

Let $Q=\left(Q_{0}, Q_{1}\right)$ be a quiver, $k$ be a field and $\mathcal{C}_{Q}$ be the category of all paths in $Q$, which is locally finite. By definition, the coalgebra $k \mathcal{C}_{Q}$ is the path coalgebra $k Q$ of the quiver.

Cibils and Rosso [6] (see also [5]) showed that a graded Hopf algebra structure on $k Q$ endows $Q_{0}$ with a group and $k Q_{1}$ with a $k Q_{0}$-Hopf bimodule structure. This in turn gives rise to what Cibils and Rosso call ramification data.

DEFINITION 19 (Ramification data). Let $G$ be a group. By ramification data for $G$, we mean a sum $r=\sum_{C \in \mathfrak{C}} r_{C} C$ for $\mathfrak{C}$ the set of conjugacy classes of $G$ and all $r_{C} \in \mathbb{N}$.

DEFINITION 20 (Hopf quiver). Let $G$ be a group and $r$ some ramification data for $G$. The quiver with $Q_{0}=G$ and $r_{C}$ arrows from $g$ to $c g$ for each $g, c \in G$, where $C$ is the conjugacy class containing $c$, is called the Hopf quiver determined by $(G, r)$.

THEOREM 4. The path coalgebra $k Q$ of a quiver $Q$ admits graded Hopf algebra structures if and only if $Q$ is a Hopf quiver.

To be more precise, for a Hopf quiver $Q$, there is a canonical Hopf algebra structure on $k Q$, with $k Q_{1} \cong \bigoplus_{C \in \mathfrak{C}^{c}} r_{C} k C \otimes k Q_{0}$ as vector space. The $k Q_{0}$-bimodule structure is given by

$$
x(c \otimes g) y=x c x^{-1} \otimes x g y,
$$

and the $k Q_{0}$-bicomodule structure is given by

$$
c \otimes g \mapsto c g \otimes(c \otimes g) \otimes g .
$$

The group structure on $Q_{0}$ and the $k Q_{0}$-bimodule structure on $k Q_{1}$ define the algebra structure on $k Q$ in lowest degrees, which is extended universally to all of $k Q$, see [6, Theorem 3.8]. However, given a Hopf quiver, there are in general also Hopf algebra structures on $k Q$ compatible with $r$ that are different from the canonical one. The choice is in the $k Q_{0}$-bimodule structure of $k Q_{1}$ which amounts to a choice of an $n_{C}$-dimensional representation of the centraliser of an element $c \in C$. The extension of the product to paths is then unique.

Huang and Torecillas [16] proved that a quiver path coalgebra $k Q$ always admits graded bialgebra structures. The results of Green and Solberg [13] are also closely related, 
but different in that they study path algebras rather than path coalgebras. Here, we focus on the Hopf algebra setting as considered in [6].

Our key aim is to stress that the product in a quiver Hopf algebra $k Q$ is not a linear extension of a monoidal product on $\mathcal{C}_{Q}$ unless $Q_{1}=\emptyset$. To do this, we classify all monoidal structures on path categories of quivers whose vertices form a group under *

LEMMA 7. Assume that the path category $\mathcal{C}_{Q}$ of a quiver is monoidal such that $\left(Q_{0}, \cdot\right)$ forms a group. Then:

(1) The monoidal product defines commuting left and right $Q_{0}$-actions on $Q_{1}$.

(2) The path length $\ell$ is a grading with respect to both $\cdot$ and $\circ$.

(3) Either $Q_{1}$ is empty, or there exists an element $z \in Z\left(Q_{0}\right)$ such that $Q_{1}$ contains for each $a \in Q_{0}$ exactly one arrow $f_{a}: a \rightarrow z \cdot a$.

Proof. If $Q_{1}=\emptyset$, there is nothing to prove, so we assume $Q_{1} \neq \emptyset$.

1. To begin with, we prove that for any identity morphism $i_{a}, a \in Q_{0}$, and any arrow $f: b \rightarrow c \in Q_{1}$, we have $i_{a} \cdot f, f \cdot i_{a} \in Q_{1}$. Indeed, we have

$$
i_{a} \cdot f=g_{1} \circ \ldots \circ g_{n},
$$

for unique arrows $g_{i} \in Q_{1}$, and then

$$
f=i_{a^{-1}} \cdot\left(g_{1} \circ \ldots \circ g_{n}\right)=\left(i_{a^{-1}} \cdot g_{1}\right) \circ \ldots \circ\left(i_{a^{-1}} \cdot g_{n}\right),
$$

where we used that $i_{a} \cdot i_{a^{-1}}=i_{a \cdot a^{-1}}=i_{1}$ and that $\cdot$ is a monoidal product. It is impossible that $i_{a^{-1}} \cdot g_{i}=i_{b}$ for some $b \in Q_{0}$, as we would then have $g_{i}=i_{a} \cdot i_{b}=i_{a \cdot b}$. So the right-hand side of the above equation is a path of length at least $n$, while the left-hand side has length 1 , hence $n=1$. Analogously, one proves $f \cdot i_{a} \in Q_{1}$. That . defines commuting actions is immediate.

2. For any arrow $f$, let $s(f), t(f)$ denote its source and target vertices, respectively. As - is a monoidal product, we have for any two arrows $f, g$ :

$$
f \cdot g=\left(i_{t(f)} \cdot g\right) \circ\left(f \cdot i_{s(g)}\right)=\left(f \cdot i_{t(g)}\right) \circ\left(i_{s(f)} \cdot g\right) .
$$

By what has been shown already, this is a path of length 2 . Continuing inductively, one proves that $\ell(f \cdot g)=\ell(f)+\ell(g)$ for all paths $f, g \in \mathcal{C}_{Q}$.

3. We also conclude from (3.3) that

$$
s(f) \cdot t(g)=t(f) \cdot s(g) \Rightarrow t(g) \cdot s(g)^{-1}=s(f)^{-1} \cdot t(f)=: z \in Q_{0}
$$

is constant (independent of $f$ ). So

$$
t(f)=s(f) \cdot z=z \cdot s(f),
$$

and any arrow $f \in Q_{1}$ with source $a$ has the same target $z \cdot a=a \cdot z$.

Given any arrow $f: a \rightarrow a \cdot z$ and $b \in Q_{0}$, there exists $i_{b} \cdot f: b \cdot a \rightarrow z \cdot b \cdot a$. This means that the same number of arrows go out of each vertex and that $z$ is in the centre of $Q_{0}$.

Finally, assume there are two arrows $f, g$ with source 1 (the unit element of $Q_{0}$ ) and consider again (3.3):

$$
f \cdot g=\left(i_{z} \cdot g\right) \circ f=\left(f \cdot i_{z}\right) \circ g .
$$

We deduce that $f=g$. 
If $Q_{1}=\emptyset$, then $\mathcal{C}_{Q}=\left\{i_{a}: a \in Q_{0}\right\}$ is just a group. The monoidal prouct is the group multiplication and this is ULF. The Hopf algebra $k \mathcal{C}_{Q}$ is the group algebra of $Q_{0}$ and is combinatorial.

If $Q_{1} \neq \emptyset$, there are two sub-cases: $z=1$ and $z \neq 1$. In the first case, each vertex $a \in Q_{0}$ has a unique arrow $f_{a}: a \rightarrow a$. In the second case, each vertex $a$ has one incoming arrow $f_{z^{-1} \cdot a}: z^{-1} a \rightarrow a$ and one outgoing arrow $f_{a}: a \rightarrow z a$.

In both cases, any morphism in $\mathcal{C}_{Q}$ can be uniquely expressed as

$$
f_{z^{n} \cdot a} \circ \ldots \circ f_{a}, \quad a \in Q_{0}, n \in \mathbb{N},
$$

to be interpreted as $i_{a}$ when $n=0$. The monoidal product is given by

$$
\left(f_{z^{n} \cdot a} \circ \ldots \circ f_{a}\right) \cdot\left(f_{z^{l} \cdot b} \circ \ldots \circ f_{b}\right)=f_{z^{n+l} \cdot a \cdot b} \circ \ldots \circ f_{a \cdot b} .
$$

This product is evidently not ULF, so we obtain:

THEOREM 5. Given a Hopf quiver $Q$, the quiver Hopf algebra $k Q$ is a case of Theorem 3 if and only if $Q_{1}=\emptyset$

REMARK 5 (Another non-example). In [8], Crossley defines several Hopf algebra structures on the vector space $k\langle S\rangle$ spanned by words on a set $S$. In two of them, the coalgebra structure is an incidence coalgebra (on the free monoidal category with one object and a morphism for each $s \in S$ ). However, just like the quiver Hopf algebras of Cibils and Rosso, these two Hopf algebras are not examples of our construction, as in both cases, $\langle S\rangle \cdot\langle S\rangle \nsubseteq\langle S\rangle$.

4. Weak Hopf algebras from monoidal categories. In this final section, we discuss the weak analogue of Theorem 3.

DEFINITION 21 (2-group). A strict 2-group is a strict monoidal groupoid in which every object is invertible, that is, in which we have $\forall x \in O b \mathcal{C} \exists y \in O b \mathcal{C}: x \cdot y=1=y \cdot x$.

LEMMA 8. Let $\mathcal{C}$ be a strict 2-group. Then for any $f \in \mathcal{C}$, there exists $\bar{f} \in \mathcal{C}$ such that $\bar{f} \cdot f=i_{1}=f \cdot \bar{f}$.

Proof. Take $\bar{f}=i_{\overline{t(f)}} \cdot f^{-1} \cdot i_{\overline{s(f)}}$, where $\overline{t(f)}$ and $\overline{s(f)}$ denote the inverses in $(O b \mathcal{C}, \cdot)$.

Strict 2-groups are the objects of a subcategory of Grpd, where we keep only the functors of groupoids which preserve the group structure of $\mathcal{C}$ with respect to $\cdot$

DEFinition 22 (Source subgroup). In any 2-group $(\mathcal{C}, \cdot, 1)$, we define the source subgroup

$$
s^{-1}(1):=\bigcup_{x \in O b \mathcal{C}} \mathcal{C}(1, x)
$$

which contains all morphisms with source 1 . This is a normal subgroup of $(\mathcal{C}, \cdot)$.

LEMMA 9. A 2-group $(\mathcal{C}, \cdot, 1)$ is locally finite if and only if $\left|s^{-1}(1)\right|$ is finite. In this case, the monoidal product has the $\left|s^{-1}(1)\right|-L F$ property as defined in Definition 5. 
Proof. Multiplication by $i_{x}$ defines a bijection $\mathcal{C}(1, y) \rightarrow \mathcal{C}(x, x y)$ for all $x, y \in O b \mathcal{C}$. As $\mathcal{C}$ is a groupoid, we have

$$
\begin{aligned}
N_{2}(f) & =\left\{\left(f \circ g^{-1}, g\right) \mid s(g)=s(f)\right\} \\
\Rightarrow\left|N_{2}(f)\right| & =|\{g \mid s(g)=s(f)\}|=\left|s^{-1}(1)\right|,
\end{aligned}
$$

for all $f \in \mathcal{C}$, and the map $N_{2}(f) \times N_{2}(g) \rightarrow N_{2}(f \cdot g)$ from Definition 5 has inverse

$$
N_{2}(f \cdot g) \ni(x, y) \mapsto\left\{((x, y),(\bar{x} \cdot a, \bar{y} \cdot b)) \mid(x, y) \in N_{2}(f)\right\} .
$$

REMARK 6. The second part of this lemma implies that a 2-group $\mathcal{C}$ is a Möbius category if and only if $\mathcal{C}=I d \mathcal{C}$.

The $\left|s^{-1}(1)\right|-$ LF property is sufficient to define compatible algebra and coalgebra structures on $k \mathcal{C}$ using the scaled version of the incidence coalgebra (Definition 3). As it is less well known than the defintion of of a Hopf algebra, we recall here the definition of a weak Hopf algebra, following [3]:

Definition 23 (Weak Hopf algebra). Let $k$ be a field. A weak $k$-Hopf algebra is a tuple $(A, \mu, 1, \Delta, \epsilon, S)$ satisfying:

(A1) $(A, \mu, 1)$ is an associative unital $k$-algebra:

(A2) $(A, \Delta, \epsilon)$ is a coassociative counital $k$-coalgebra

(A3) The coproduct is multiplicative: $\Delta(a b)=\Delta(a) \Delta(b)$

(A4) The counit is weakly multiplicative:

$$
\epsilon(a b)=\epsilon\left(a 1_{(1)}\right) \epsilon\left(1_{(2)} b\right)=\epsilon\left(a 1_{(2)}\right) \epsilon\left(1_{(1)} b\right),
$$

(A5) The unit is weakly comultiplicative:

$$
(\Delta(1) \otimes 1)(1 \otimes \Delta(1))=\Delta^{2}(1)=(1 \otimes \Delta(1))(\Delta(1) \otimes 1),
$$

(A6) $S: A \rightarrow A$ is a $k$-linear map (called the antipode) which satisfies

$$
\begin{aligned}
S(a) & =S\left(a_{(1)}\right) a_{(2)} S\left(a_{(3)}\right) \\
S\left(a_{(1)}\right) a_{(2)} & =\epsilon\left(1_{(1)} a\right) 1_{(2)} \\
a_{(1)} S\left(a_{(2)}\right) & =1_{(2)} \epsilon\left(a 1_{(2)}\right) .
\end{aligned}
$$

REMARK 7. Under the assumption of axioms (A1), (A2) and (A3), the version of axiom (A4) given here is equivalent to the formulation in [3]:

$$
\epsilon(a b c)=\epsilon\left(a b_{(1)}\right) \epsilon\left(b_{(2)} c\right)=\epsilon\left(a b_{(2)}\right) \epsilon\left(b_{(1)} c\right) .
$$

We can now state the central theorem of this section:

THEOREM 6. Let $(\mathcal{C}, \cdot, 1)$ be a locally finite 2-group, $k$ a field of characteristic zero and $k \mathcal{C}$ the $k$-vector space spanned by the morphisms of $\mathcal{C}$. If $(k \mathcal{C}, \Delta, \varepsilon)$ is the scaled incidence coalgebra of $k \mathcal{C}$ with $\lambda=\left|s^{-1}(1)\right|$ (Definition 3), then $\left(k \mathcal{C}, \cdot, i_{1}, \Delta, \epsilon, S\right)$ is a weak Hopf algebra with antipode $S(f)=\bar{f}^{-1}$.

Proof. (A1) and (A2) are immediate by Theorems 2 and 3. (A3) then follows by Lemma 9 and (A4) by Lemma 8. For (A5), we observe that

$$
\begin{array}{r}
N_{2}\left(i_{1}\right) \times N_{2}\left(i_{1}\right) \rightarrow N_{3}\left(i_{1}\right) \\
((a, b) ;(c, d)) \mapsto(a, b \cdot c, d),
\end{array}
$$


defines a bijection and that $b \circ c=b \cdot c=c \cdot b$ for $s(b)=t(c)=1$. (A6) can be verified by a short computation.

4.1. Example: Relations on groups. Here, we present the analogy to Section 3.1: let $\sim$ be a reflexive transitive relation on a group $G$ which is compatible with the multiplication as in (3.1) and consider the category $\mathcal{C}$ with $O b \mathcal{C}=G, \mathcal{C}(h, g)=\{(h, g) \mid g \sim h\}$. This category is a locally finite 2 -group iff $\sim$ is an equivalence relation and the equivalence classes are finite.

By Theorem 6, $k \mathcal{C}$ admits a weak incidence Hopf algebra structure with a coproduct which runs through equivalence classes:

$$
\Delta(h, g)=\frac{1}{|N|} \sum_{g \sim f \sim h}(h, f) \otimes(f, g),
$$

where $N=\{g \in G \mid g \sim 1\}$. In the case that $\sim$ is equality, the weak Hopf algebra obtained is the group algebra $k G$. This can be alternatively stated in the following way: let $G$ be a group and $N \triangleleft G$ a normal subgroup. Define a 2-group $\mathcal{C}$ by $O b \mathcal{C}=G$ and

$$
|\mathcal{C}(g, h)|=\left\{\begin{array}{l}
1, \exists z \in N: h=z g \\
0, \text { else }
\end{array} .\right.
$$

Then, $k \mathcal{C}$ admits a weak incidence Hopf algebra if and only if $|N|$ is finite.

4.2. Example: Automorphism 2-groups. Let $\mathcal{C}=\left(\mathrm{ObC} \mathrm{Mor}_{1} \mathcal{C}, \mathrm{Mor}_{2} \mathrm{C}\right)$ be a strict 2-category. Then, for each $x \in O b \mathcal{C}$, there exists a strict 2-group AUT $(x)$ whose objects are the automorphisms of $x$ as an object of $\mathcal{C}$ and whose morphisms are the 2-isomorphisms between these. The product of two objects $f, f^{\prime}$ in $\operatorname{AUT}(x)$ is given by their composition in $\mathcal{C}$ and the unit object is $i_{x}$. The composition of morphisms in $\operatorname{AUT}(x)$ is given by the vertical composition of 2-morphisms in $\mathcal{C}$, and the product of morphisms in $\operatorname{AUT}(x)$ is given by the horizontal composition in $\mathcal{C}$. According to Theorem $6, k \mathrm{AUT}(x)$ admits a weak incidence Hopf algebra structure iff $\left\{f \in \operatorname{Mor}_{1} \mathcal{C} \mid f \sim i_{x}\right\}$ is finite.

This example overlaps with Example 4.1. Consider the 2-category Grp, whose objects are groups, 1-morphisms are group homomorphisms and 2-morphisms are given by inner automorphisms in the target group, that is, for $f_{1}, f_{2}: G \rightarrow H$, there exists $\phi_{h}: f_{1} \rightarrow f_{2}$ iff there exists $h \in H$ such that $f_{1}=f_{h} \circ f_{2}$, where $f_{h}$ is the morphism in $\operatorname{Inn}(H)$ given by conjugation by $h$. If $G$ is an object in $\operatorname{Grp}, \operatorname{ObAUT}(G):=\operatorname{Aut}(G)$, the usual group of automorphisms of $G$, and $\operatorname{AUT}(G)$ (the set of morphisms) contains an arrow between any pair of autmorphisms which are related by an inner automorphism of $G$. Hence, $k \operatorname{AUT}(G)$ admits a weak Hopf algebra structure iff $\operatorname{Inn}(G)$ is finite.

4.3. Crossed modules. Here, we will reformulate the result of Theorem 6 in the language of crossed modules.

Definition 24 (Crossed module). A crossed module $(G, H, \tau, \alpha)$ consists of:

(1) Groups $G, H$

(2) A group action $\alpha: G \times H \rightarrow H$

(3) A group homomorphism $\tau: H \rightarrow G$.

such that $\alpha$ and $\tau$ satisfy 


$$
\begin{aligned}
\tau(\alpha(g, h)) & =g \tau(h) g^{-1} \\
\alpha\left(\tau(h), h^{\prime}\right) & =h h^{\prime} h^{-1} .
\end{aligned}
$$

Crossed modules form the objects of a category whose morphisms $\left(f_{1}, f_{2}\right)$ : $(G, H, \tau, \alpha) \rightarrow\left(G^{\prime}, H^{\prime}, \tau^{\prime}, \alpha^{\prime}\right)$ consist of pairs of group homomorphisms $f_{1}: G \rightarrow G^{\prime}$ and $f_{2}: H \rightarrow H^{\prime}$ such that $\tau^{\prime} f_{2}=f_{1} \tau$.

THEOREM 7 (Brown and Spencer). The categories of strict 2-groups and crossed modules are equivalent.

For the original statement and proof, see [4], and for further exploration, see[1, 10].

Given a 2 -group $(\mathcal{C}, \cdot, 1)$, the corresponding crossed module $(G, H, \alpha, \tau)$ is defined as follows: set $G=O b \mathcal{C}, H=s^{-1}(1), \alpha(g, h)=i_{g} \cdot h \cdot i_{g^{-1}}$ and $\tau(h)=t(h)$.

Conversely, given a crossed module $(G, H, \alpha, \tau)$, the corresponding strict 2-group $(\mathcal{C}, \cdot, 1)$ has $O b \mathcal{C}=G, \mathcal{C}=H \rtimes_{\alpha} G, s(h, g)=g$ and $t(h, g)=\tau(h) g$. Composition of morphisms is given by $\left(h^{\prime}, \tau(h) g\right) \circ(h, g)=\left(h^{\prime} h, g\right)$.

This equivalence gives us the following:

COROLlary 1 (to Theorem 6). Let $(G, H, \tau, \alpha)$ be a crossed module, $k$ a field and $k(H \times G)$ the free $k$-module on the set $H \times G$. Then $k(H \times G)$ admits the following weak Hopf algebra structure iff $|H|$ is finite:

$$
\begin{aligned}
(h, g) \cdot\left(h^{\prime}, g^{\prime}\right) & =\left(h \alpha\left(g, h^{\prime}\right), g g^{\prime}\right) \\
\Delta(h, g) & =\sum_{h^{\prime} h^{\prime \prime}=h}\left(h^{\prime \prime}, \tau\left(h^{\prime}\right) g\right) \otimes\left(h^{\prime}, g\right) \\
S(h, g) & =\left(\alpha\left(g^{-1}, h^{-1}\right)^{-1}, g^{-1}\right) .
\end{aligned}
$$

If $\tau: H \rightarrow G$ is injective, we are in the case of Example 4.1, that is, $H$ is isomorphic to a normal subgroup of $G$.

ACKNOWLEDGements. It is our pleasure to thank Gabriella Böhm, Joachim Kock and Michele Sevegnani for discussions and suggestions, as well as the referees for their careful reading and valuable input.

\section{REFERENCES}

1. J. C. Baez and A. D. Lauda, Higher-dimensional algebra V: 2-groups, Theory Appl. Categor. 12 (2004), 423-491.

2. C. Bergbauer and D. Kreimer, Hopf algebras in renormalisation theory: locality and DysonSchwinger equations from Hochschild cohomology, IRMA Lect. Math. Theor. Phys. 10 (2006), 133-164.

3. G. Böhm, F. Nill and K. Szlachányi, Weak Hopf algebras I. Integral theory and $C^{*}$-structure, arXiv:math/9805116.

4. R. Brown and C. B. Spencer, G-groupoids, crossed modules, and the classifying space of a topological group, Proc. Kon. Akad. v. Wet. 79 (1976), 296-302.

5. C. Cibils, A. Lauve and S. Witherspoon, Hopf quivers and Nichols algebras in positive characteristic (2009).

6. C. Cibils and M. Rosso, Hopf quivers (2000), arXiv:math/0009106.

7. A. Connes and D. Kreimer, Hopf algebras, renormalization and noncommutative geometry, Commun. Math. Phys. 199 (1998), 203-242.

8. M. D. Crossley, Some Hopf algebras of words, Glasgow Math. J. 48 (2006), 575-582.

9. L. Foissy, An introduction to Hopf algebras of trees, preprint. 
10. M. Forrester-Barker, Group objects and internal categories (2002), arXiv:math/0212065v1.

11. I. Gàlvez-Carillo, J. Kock and A. Tonks, Decomposition spaces in combinatorics (2016), arXiv: $1612.09225 \mathrm{v} 2$.

12. I. Gàlvez-Carillo, J. Kock and A. Tonks, Decomposition Spaces, incidence algebras and Möbius inversion III: the decomposition space of Möbius intervals (2015), arXiv:1512.07580.

13. E. L. Green and Ø. Solberg, Basic Hopf algebras and quantum groups, Mathematische Zeitschrift 229(1) (1998), 45-76.

14. D. Grinberg and V. Reiner, Hopf algebras in combinatorics (2014), arXiv:1409.8356.

15. R. Holtkamp, On Hopf algebra structures over free operads, Adv. Math. 207(2) (2006), 544-565.

16. H.-L. Huang and B. Torrecillas, Quiver bialgebras and monoidal categories, Colloq. Math. 131 (2013), 287-300.

17. O. H. Jensen and R. Milner, Bigraphs and mobile processes (revised), Technical Report (University of Cambridge computer laboratory, 2004).

18. S. A. Joni and G.-C. Rota, Coalgebras and bialgebras in combinatorics, Stud. Appl. Math. 61 (1979), 93-139.

19. R. M. Kaufmann and B. C. Ward, Feyman categories, arXiv:1312.1269v3.

20. J. Kock, Polynomial functors and combinatorial Dyson-Schwinger equations, J. Math. Phys. 58 (2017), 041703.

21. F. W. Lawvere and M. Menni, The Hopf algebra of Möbius intervals, Theory Appl. Categor. 24(10) (2010), 221-265.

22. P. Leroux, Les Categories de Möbius, Cahiers de Topologie et Gèometrie Diffèrentielle Catègoriques 16(3) (1975) 280-282.

23. T. Leinster, Notions of Möbius inversion, Bull. Belg. Math. Soc. Simon Stevin 19(5) (2012), 911-935.

24. J.-L. Loday and M. Ronco, Combinatorial Hopf algebras (2008), arXiv:0810.0435.

25. D. Manchon, Hopf algebras, from basics to applications to renormalization, Comptes Rendus des Rencontres Mathematiques de Glanon (2001).

26. R. Milner, The space and motion of communicating agents (Cambridge University Press, 2008).

27. D. E. Radford, Hopf algebras (World Scientific Publishing Europe Ltd, UK, 2012).

28. L. Rotheray, Hopf Subalgebras from Green's Functions MSc Thesis (Humboldt-Universität zu Berlin, 2015).

29. M. Sevegnani and M. Calder, Bigraphs with sharing, TR-2010-310, DCS Technical Report Series (Department of Computing Science, University of Glasgow, 2010).

30. K. Yeats, A Hopf algebraic approach to Schur function identities, arXiv:1511.06337v4. 